\title{
Distribución del cedro rojo (Cedrela odorata L.) en el estado de Hidalgo, bajo condiciones actuales y escenarios de cambio climático \\ Distribution of red cedar (Cedrela odorata L.) in the Hidalgo state, under current conditions and scenarios of climate change
}

\author{
Jesús David Gómez Díaz ${ }^{1}$, Alejandro Ismael Monterroso Rivas ${ }^{1}$ \\ y Juan Ángel Tinoco Rueda ${ }^{1}$
}

\section{RESUMEN}

Se obtuvieron las áreas climáticas potenciales de distribución del cedro rojo (Cedrela odorata L.) en el estado de Hidalgo bajo condiciones actuales, con información meteorológica de 1961-1990 como escenario base, y bajo escenarios de cambio climático obtenidos con los modelos GFDL-R30 y el HadCM3 para los años 2020 y 2050. Se aplicaron las tasas de cambio en temperatura y precipitación sobre las áreas de influencia climática delimitadas de acuerdo a Gómez et al. (2006). Se realizó un balance de humedad bajo condiciones actuales y de cambio climático aplicando la metodología de Thornthwaite modificada versión III (Monterroso y Gómez, 2003). Actualmente se reporta 9,8\% de la superficie del estado con tipos de vegetación con los que se asocia el cedro rojo, y las estimaciones con las características climáticas del escenario base muestran que el $30,4 \%$ del estado presenta algún grado de aptitud para el desarrollo de esta especie. Los resultados del modelo GFDL-R30 indican un incremento en la superficie con algún nivel de aptitud, con respecto al escenario base, de 3,1\% y $4,4 \%$ para el año 2020 y 2050, respectivamente. El aumento es diferencial dentro de las clases de aptitud. Para el modelo HadCM3 la superficie con algún nivel de aptitud disminuye 0,9\% para el año 2020 y $0,2 \%$ para el año 2050 . Sin embargo, el nivel de Moderadamente apto pasa de $10,5 \%$ en el escenario base a $0 \%$ y $1,3 \%$ para el año 2020 y 2050 , respectivamente, ubicándose practicamente todas las áreas en el menor nivel de aptitud.

PALABRAS CLAVE:

Balance de humedad, cambio climático, cedro rojo, zonificación agroclimática.

\section{ABSTRACT}

The potential climatic areas for the distribution of red cedar (Cedrela odorata L.) were obtained in the state of Hidalgo under actual conditions, with meteorological information of 1961-1990 as base line, and under climate change conditions wich were obtained with two models: GFDL-R30 and HadCM3 for two time scenarios (2020 and 2050). The adjustment rates in temperature and rainfall obtained for each model were applied on climatic influence areas delimitated according to Gómez et al. (2006). A soil moisture balance in the state's surface was calculated under current and climate change conditions using the Thornthwaite, modified version III methodology (Monterroso and Gómez, 2003). The present percentage of the state surface with the vegetation types associated with red cedar is of $9,8 \%$ and the surface percentage estimated with some degree of suitability for this specie, using the climatic characteristics of the baseline scenario, is of $30,4 \%$. The results of applied GFDL-R30 model shows an increment on the total surface with some degree of suitability with respect of the baseline scenario of $3,1 \%$ and $4,4 \%$ for the

1 Universidad Autónoma Chapingo, Departamento de Suelos, Km. 38.5 carretera México-Texcoco. Chapingo. México, C.P. 56230. dgomez@correo.chapingo.mx 
years 2020 and 2050, respectively, with a differential increment within the suitability classes. The surface estimated with some degree of suitability applying the HadCM3 model shows a decrement of $0,9 \%$ for the year 2020 and $0,2 \%$ for 2050 . However, the class of Moderate suitable, change from $10,5 \%$ on the baseline scenario to $0 \%$ and $1,3 \%$ for the years 2020 and 2050, respectively, with almost all the areas in the lowest level of suitability.

KEY WORDS:

Water balance, climate change, red cedar, agro climatic zoning.

\section{INTRODUCCIÓN}

La especie objeto de estudio en este trabajo, el cedro rojo (Cedrela odorata L.), se localiza primordialmente en ecosistemas húmedos, en específico en el bosque tropical perennifolio, el bosque tropical subcaducifolio, el bosque tropical caducifolio y el bosque mesófilo de montaña (CONAFOR, 2000). Partiendo de esta información y de la distribución reportada en el inventario nacional forestal para estos tipos de vegetación (SEMARNAT-UNAM, 2001), se estima que el cedro rojo en el estado de Hidalgo, se encuentra distribuido en áreas fragmentadas en la zona de barlovento que comprende parte de la planicie costera del Golfo norte, los flancos al este de la Sierra Madre Oriental hasta alturas intermedias menores a los 2000 msnm. La superficie de los tipos de vegetación con los que se asocia el cedro rojo cubren $2040,3 \mathrm{~km}^{2}$ lo que equivale al $9,8 \%$ del territorio total del estado de Hidalgo.

La distribución de las comunidades vegetales está determinada por diferentes factores ambientales como las características del suelo y el clima; siendo este último el principal responsable (Zunino y Zullini, 2003; Berry et al., 2002). Cuando alguno de dichos factores cambia, en especial las condiciones climáticas, la estructura y composición de los ecosis- temas también pueden sufrir modificaciones. La respuesta por parte de las plantas y animales depende de la intensidad y velocidad del cambio en las condiciones climáticas, si el cambio es paulatino y con poca intensidad, es probable que gran parte de las especies logre evolucionar y sus descendientes se adapten a las nuevas condiciones ambientales. Sin embargo, si el cambio se produce con gran intensidad y en un periodo relativamente corto, un bajo porcentaje de la biodiversidad logra adaptarse a estos cambios, lo que produce la extinción de las especies con baja capacidad de amortiguamiento. Esta capacidad de adaptación de las especies se ha venido presentando desde la aparición de la vida sobre el planeta Tierra (Pérez, 2005).

La temperatura y la precipitación son dos de los elementos climáticos determinantes para el establecimiento de una especie y/o una comunidad vegetal en determinado sitio (Velázquez, 2002). Por su parte, Ortiz (1987) establece que la mayoría de los procesos fisiológicos que se realizan para el crecimiento y desarrollo de las plantas están fuertemente influenciados por la temperatura. En términos generales, ésta ejerce su influencia principal controlando la proporción de reacciones químicas involucradas en varios procesos de crecimiento dentro de la planta. La temperatura, además, afecta los mecanismos hormonales involucrados en la floración y fructificación de las plantas.

El agua por su parte, resulta indispensable para la existencia y evolución de los ecosistemas vegetales y animales. Es un elemento asociado a condiciones climáticas y edafológicas, por lo que su cantidad en la atmósfera y en el suelo tiene una repercusión directa en los ecosistemas. El agua es la parte esencial del protoplasma vegetal, constituye un elemento importante de la fotosíntesis y un medio indispensable de suministro de elementos nutritivos. En 
forma de vapor, el efecto principal del agua consiste en dar humedad al aire; un alto contenido de humedad del aire reduce la evaporación de los suelos así como la evapotranspiración de las plantas (Aguilera y Martínez, 1996).

Los cambios en el sistema climático global han ocurrido durante toda la historia del planeta. Dichas modificaciones se han presentado por causas naturales como por ejemplo: cambios en la órbita terrestre, alteraciones en la excentricidad del planeta, actividad volcánica intensa e impacto de meteoritos (Rivera, 1999). Sin embargo, desde hace varias décadas se ha incluido al hombre como una de las causas de cambio climático. Tal cambio le es atribuido directa o indirectamente a las actividades humanas que alteran la composición de la atmósfera, que agregadas a la variabilidad climática natural observada en periodos comparables de tiempo, han creado un fuerte consenso científico de que el clima global se verá alterado significativamente en este siglo, como resultado del aumento de la concentración de Gases de Efecto Invernadero (GEI), tales como el dióxido de carbono $\left(\mathrm{CO}_{2}\right)$, el metano $\left(\mathrm{CH}_{4}\right)$, los óxidos de nitrógeno $\left(\mathrm{N}_{2} \mathrm{O}\right)$ y los clorofluorocarbonos (CFCs) (IPCC, 2001). La concentración de gases de efecto invernadero en la atmósfera continúa incrementándose, provocando en general un efecto de calentamiento. Aunque en algunas regiones hay también un incremento en la concentración de aerosoles, éstos tienen un efecto opuesto en el balance de la radiación, esto es, tienden a enfriar. Los cambios en la concentración de GEI y aerosoles son los que causan cambios regionales y globales en la temperatura, precipitación y otras variables climáticas. Esto implica cambios globales en la humedad del suelo, incrementos en el nivel del mar y la proyección de eventos más extremosos en cuanto a temperaturas elevadas, inundaciones $y$ sequías en una gran mayoría de localidades (Houghton et al., 1996). EI IPCC (Intergovernmental Panel on Climate Change) establece que las concentraciones de $\mathrm{CO}_{2}$ durante el periodo 17502000 aumentaron en un $31 \%$, debido principalmente a la quema de combustibles fósiles y al cambio del uso del suelo (IPCC, 2002).

A consecuencia de la ocurrencia de eventos extremos, centros de investigación y universidades de todo el mundo se han dado a la tarea de crear herramientas para la simulación de los impactos que los cambios en el clima podrían ocasionar a los ecosistemas y por consecuencia a las actividades humanas. Para estimar las condiciones futuras del clima con base en las nuevas concentraciones de los gases de efecto invernadero se han desarrollado diferentes modelos que simulan los cambios en las variables climáticas como por ejemplo la temperatura, precipitación, evaporación, etc. (Magaña et al., 2000).

A partir de la aplicación de los modelos de cambio climático se obtienen las variables base para realizar simulaciones a futuro del comportamiento de especies, de ecosistemas o bien de sistemas de producción agrícola, ganadero o silvícola como es el caso de las investigaciones realizadas por Bakkenes et al. (2006); Berry et al. (2002); Yáñez et al. (1998); Olfert y Weiss (2006); Herrmann et al. (2005), así como Hitz y Smith (2004).

Como se observa, es importante profundizar en estudios sobre los impactos que un posible cambio en las condiciones globales climáticas tendrían sobre los ecosistemas del país y de una manera más regional, con motivo de apoyar los criterios de planeación que actualmente se llevan a cabo en programas nacionales tales como el Programa Nacional de Reforestación 
(PRONARE), Programa de Desarrollo Forestal (PRODEFOR) y el Programa de Plantaciones Forestales Maderables (PRODEPLAN), entre otros.

\section{OBJETIVOS}

1. Elaborar cartografía actualizada de las variables climáticas, temperatura y precipitación del estado de Hidalgo con datos de 1961 a 1990.

2. Realizar un balance de humedad en el estado de Hidalgo bajo condiciones actuales y en escenarios de cambio climático con la aplicación de dos modelos, el norteamericano (GFDL-R30) y el inglés (HadCM3), para evaluar los posibles cambios en la disponibilidad de humedad en el suelo que afecta la distribución del cedro rojo.

3. Comparar la distribución actual del cedro rojo (Cedrela odorata L.), su distribución potencial actual, así como la distribución potencial bajo escenarios de cambio climático con la aplicación de los dos modelos señalados.

\section{METODOLOGÍA}

\section{Área de estudio}

El estado de Hidalgo es una de las 31 entidades federativas de la República Mexicana, se localiza en la zona centro del país, entre las coordenadas máximas: $19^{\circ} 36^{\prime} 36^{\prime \prime}$ y $21^{\circ} 24^{\prime} 36^{\prime \prime}$ de latitud norte y $97^{\circ} 57^{\prime}$ y $99^{\circ} 50^{\prime} 24^{\prime \prime}$ de longitud oeste. Presenta un amplio rango altitudinal que va de los 100 hasta los 3400 metros sobre el nivel del mar. Con base en el inventario nacional forestal del año 2001 (SEMARNAT-UNAM, 2001), la agricultura es el principal uso de la tierra ocupando $47 \%$ de la superficie estatal, seguido por el pastizal inducido con $15 \%$. Se presenta una gran diversidad de ecosistemas siendo los bosques templados los de mayor distribución con el $26 \%$ del territorio estatal.

\section{Delimitación de áreas de influencia climática}

Para la búsqueda de información climática inicialmente se ubicaron las estaciones climatológicas que se encuentran dentro de los límites y en la periferia del estado de Hidalgo. Posteriormente se utilizaron las normales climatológicas publicadas por el Servicio Meteorológico Nacional (SMN, 2000) para la extracción de los datos promedios mensuales y anual de precipitación y temperatura para el periodo de 1961-1990.

Para el trazo de las isoyetas se utilizó la metodología propuesta por Gómez et al. (2006), en la cual se emplea como información base a espaciomapas y/o imágenes de satélite, modelos digitales de elevación, cartas topográficas en formato digital y los datos de precipitación de las estaciones meteorológicas. Utilizando el software de sistemas de información geográfica ArcView v.3.2 (ESRI, 1996) se sobrepusieron estos productos cartográficos y se trazaron las isoyetas tomando en cuenta la dinámica de circulación de los vientos, el efecto que tienen los sistemas orográficos en la dinámica de movimiento de los sistemas de vientos y su impacto en la precipitación, así como los diversos fenómenos meteorológicos que afectan al estado de Hidalgo como nortes y huracanes (Vidal, 2005). Se establecieron analogías entre la cantidad de precipitación y la distribución de la vegetación. Lo anterior para conformar el mapa de precipitación media anual y las precipitaciones mensuales.

Para obtener las isotermas se dividió al estado en dos zonas determinadas por el efecto de las barreras orográficas en la 
humedad dominante en el aire: barlovento y sotavento. Así, la vertiente que ve hacia el Golfo de México quedó dentro del área denominada barlovento y la vertiente interior del estado en el sotavento. La línea divisoria se estableció en la isoyeta anual de $1000 \mathrm{~mm}$. Para cada zona se generaron modelos de regresión lineal simple para establecer el rango de variación de la temperatura con la altura, obteniéndose doce modelos mensuales y uno anual para cada área delimitada (Gómez et al., 2006). Aplicando los modelos obtenidos se trazaron las isolíneas de temperatura por cada rango altitudinal, conformando así los doce mapas de temperatura mensual y el anual en cada área. Se unieron posteriormente, para hacer las correcciones en las áreas de confluencia cuando se presentaron diferencias de temperatura.

Finalmente, se obtuvieron las áreas de influencia climática (AIC) al sobreponer, mediante el programa de sistemas de información geográfica ArcView v.3.2 (ESRI, 1996) las isoyetas con las isotermas anuales. A cada una de las AIC resultantes se le atribuyó su correspondiente base de datos de temperatura y precipitación mensual.

Aplicación de las razones de cambio estimadas con los Modelos de Cambio Climático

Las AIC obtenidas se sobrepusieron con las zonas de cambio de temperatura y precipitación obtenidas por el Canadian Institute for Climate Studies (CICS, 2006) para el modelo de cambio climático GFDL-R30 desarrollado por el Geophysical Fluid Dynamics Laboratory, de los Estados Unidos de Norteamérica y el modelo HadCM3 desarrollado por el Hadley Centre for Climate Prediction and Research ubicado en el Reino Unido. Los modelos se evaluaron para los años 2020 y 2050.
Requerimientos climáticos que determinan la distribución del cedro rojo (Cedrela odorata L)

La matriz de los requerimientos climáticos para la distribución de Cedrela odorata L. fue una compilación de información de diferentes fuentes oficiales, siendo la principal la ficha técnica del SIRE (Sistema de Información para la Reforestación) distribuida por la Comisión Nacional Forestal (CONAFOR, 2000) así como de la base de datos de Especies Nativas Valiosas para la Reforestación (Vázquez et al., 1999) distribuida por la Comisión Nacional para el Conocimiento y Uso de la Biodiversidad (CONABIO) para esta especie.

\section{Distribución actual y potencial del cedro rojo}

La distribución actual del cedro rojo se estimó con base en los reportes de la CONAFOR (2000). Estos indican que esta especie se localiza primordialmente en ecosistemas húmedos, en específico, en el bosque tropical perennifolio, el bosque tropical subcaducifolio, el bosque tropical caducifolio y el bosque mesófilo de montaña.

La superficie en la que actualmente se distribuye se estimó a partir de la información del inventario nacional forestal del año 2001 (SEMARNAT-UNAM, 2001) en donde se presentan los tipos de vegetación con los que se asocia al cedro rojo.

Para delimitar la distribución potencial de esta especie, se determinaron las condiciones climáticas en las que es posible que prospere considerando cuatro niveles de aptitud: 1) Apto, 2) Moderadamente Apto, 3) Marginalmente Apto y 4) No Apto. 


\section{Simulación de la distribución del cedro rojo con cambio climático}

Para delimitar las posibles zonas en donde se distribuirá el cedro rojo, se identificaron los valores de temperatura y precipitación modificados por los dos modelos de cambio climático de las áreas de influencia que correspondieran con los rangos de distribución encontrados.

Considerando la distribución actual y potencial de Cedrela odorata L. y bajo escenarios de cambio climático, se obtuvieron seis escenarios de comportamiento del cedro rojo: 1) el actual o base usando la información reportada por el inventario nacional forestal (SEMARNAT-UNAM, 2001); 2) el potencial de acuerdo a las condiciones actuales; 3) y 4) el potencial futuro de acuerdo al modelo norteamericano de cambio climático a los años 2020 y 2050 y 5) y 6) la posible distribución de acuerdo al modelo inglés de cambio climático para los años 2020 y 2050.

\section{Balance de humedad en el estado de Hidalgo}

Para evaluar los cambios en la distribución de Cedrela odorata L. por efecto del cambio climático se realizó un balance de humedad en el estado de Hidalgo para los escenarios descritos $(2,3,4,5$ y 6$)$ y así identificar los posibles cambios en la cantidad de humedad disponible en el suelo. El balance se realizó utilizando la metodología de Thornthwaite modificada versión III (Monterroso y Gómez, 2003). Esta metodología fue creada en la década de los 90 por países de la Unión Europea para contar con mayor información sobre el impacto del cambio climático en sus regiones. También fue incorporada por la Convención de las Naciones Unidas de Lucha Contra la Desertificación en Países Afectados por Sequía Grave y Desertificación, para delimitar las zonas áridas y semiáridas bajo un mismo criterio (Monterroso y Gómez, 2004).
El sistema incluye la aplicación del concepto del balance hídrico. Este consiste en la relación entre el agua aportada por la precipitación y la requerida por la vegetación en sus funciones de evaporación y transpiración (Dunne y Leopold, 1978). El sistema de Thornthwaite considera un nuevo concepto en la clasificación climática, como es la eficiencia de los parámetros meteorológicos de precipitación y temperatura. La evaluación del primero se determina a través de un balance de humedad con la finalidad de determinar qué tan seco o qué tan húmedo es el clima en relación con el crecimiento de las plantas. En la evaluación del segundo parámetro se estima qué tan caliente o qué tan frío es el clima para el crecimiento de las plantas. El sistema considera además la capacidad de almacenamiento de humedad del suelo, que es la cantidad máxima de agua disponible en las capas del suelo ocupadas por las raíces y que puede ser tomada por las mismas cuando la planta lo requiera para realizar sus funciones. En su estimación se considera la cantidad de humedad a partir de la situación del punto de marchitez permanente hasta capacidad de campo. En la Tabla 1 se presenta la descripción de los tipos de climas de la metodología de Thornthwaite modificada, resultantes del balance de humedad.

\section{RESULTADOS}

\section{Delimitación de áreas de influencia climática.}

Para el trazo de las isotermas, se estimaron inicialmente los modelos de regresión lineal simple que se presentan en la Tabla 2 en donde se observa que los valores del coeficiente de regresión $\left(R^{2}\right)$ son altos, mayores de 0,9 para la zona de barlovento y mayores de 0,8 para sotavento. 
Tabla 1. Descripción de los tipos de climas usados en la metodología. Thornthwaite modificada versión III

\begin{tabular}{lccc}
\hline \multicolumn{1}{c}{ CLIMA } & $\begin{array}{c}\text { PERIODO DE } \\
\text { LLUVIAS }\end{array}$ & $\begin{array}{c}\text { DÉFICIT DE } \\
\text { HUMEDAD }\end{array}$ & $\begin{array}{c}\text { DEMASÍAS DE } \\
\text { AGUA }\end{array}$ \\
\hline Árido (E) & $<3$ meses & Muy severo & No existen \\
\hline Semiárido Seco (D1) & 3 meses & Severo & Mínimas \\
\hline Semiárido Moderado (D2) & 3 a 4 meses & Severo & Mínimas \\
\hline Semiárido Húmedo (D3) & 3 a 5 meses & No tan severo & Mínimas \\
\hline Subhúmedo Seco (C1) & 4 a 5 meses & Reducido & Moderadas \\
\hline Subhúmedo Húmedo (C2) & 4 a 6 meses & Mínimo & Mayores \\
Húmedo (B) & $>5$ meses & Mínimo & Mayores \\
Per Húmedo (A) & $>6$ meses & No existe & Mayores \\
\hline
\end{tabular}

Fuente: Monterroso y Gómez, 2003

Tabla 2. Modelos de regresión lineal simple para temperatura $(T)$ y la altura $(\mathrm{h})$ en la zona de barlovento y sotavento en el estado de Hidalgo

\begin{tabular}{|c|c|c|c|c|}
\hline \multirow{2}{*}{ MES } & \multicolumn{2}{|c|}{ BARLOVENTO } & \multicolumn{2}{|l|}{ SOTAVENTO } \\
\hline & MODELO & $\mathrm{R}^{2}$ & MODELO & $\mathrm{R}^{2}$ \\
\hline ENERO & $T=-0,0036 h+18,86$ & 0,97 & $T=-0,0049 h+22,90$ & 0,82 \\
\hline FEBRERO & $T=-0,0038 h+20,12$ & 0,96 & $T=-0,0053 h+24,78$ & 0,83 \\
\hline MARZO & $T=-0,0041 \mathrm{~h}+23,56$ & 0,94 & $T=-0,006 h+29,07$ & 0,87 \\
\hline ABRIL & $T=-0,0049 h+26,89$ & 0,97 & $T=-0,0063 \mathrm{~h}+31,40$ & 0,87 \\
\hline MAYO & $T=-0,0055 h+28,74$ & 0,97 & $T=-0,0068 h+33,38$ & 0,88 \\
\hline JUNIO & $T=-0,0063 h+29,18$ & 0,98 & $T=-0,0067 \mathrm{~h}+32,21$ & 0,86 \\
\hline JULIO & $T=-0,0062 h+28,38$ & 0,97 & $T=-0,0067 h+31,33$ & 0,86 \\
\hline AGOSTO & $T=-0,0063 h+28,46$ & 0,98 & $T=-0,0068 \mathrm{~h}+31,72$ & 0,86 \\
\hline SEPTIEMBRE & $T=-0,006 h+27,76$ & 0,98 & $T=-0,0063 h+30,18$ & 0,86 \\
\hline OCTUBRE & $T=-0,0056 h+25,45$ & 0,98 & $T=-0,0058 \mathrm{~h}+27,80$ & 0,84 \\
\hline NOVIEMBRE & $T=-0,0049 h+22,84$ & 0,97 & $T=-0,0055 h+25,71$ & 0,85 \\
\hline DICIEMBRE & $T=-0,0042 h+20,69$ & 0,95 & $T=-0,0052 h+24,06$ & 0,85 \\
\hline ANUAL & $T=-0,0051 \mathrm{~h}+25,07$ & 0,97 & $T=-0,006 h+28,72$ & 0,89 \\
\hline
\end{tabular}

En el mapa que contiene los rangos de altura se digitalizó la información obtenida de los rangos altitudinales de variación de la temperatura de los modelos de regresión lineal. Con esto se obtuvieron gráficamente las isotermas mensuales y anuales para todo el estado (Figura 1a).

Las regiones donde se presentan las temperaturas más altas, son la zona de barlovento asociada a la planicie costera y zona baja de la Sierra Madre Oriental, así como partes bajas de las cañadas de los ríos que desembocan en el Golfo de México, con valores medios anuales de 23 a $27^{\circ} \mathrm{C}$. En las partes bajas de la barranca de Metztitlán y la parte baja del Valle del Mezquital en la zona de Ixmiquilpan, los rangos de temperatura media anual son también altos y van de 17 a 
$23^{\circ} \mathrm{C}$. Por otro lado, las áreas con mayor elevación como la caldera de Huichapan, las montañas de Ixmiquilpan-Zimapan y la región sureste del estado son las que presentan las temperaturas más bajas con valores medios anuales de 9 a $11^{\circ} \mathrm{C}$.

La Figura 1b muestra que la parte más húmeda del estado se presenta en las regiones noreste y este a lo largo de la zona de barlovento de la Sierra Madre Oriental con valores medios anuales de 1900 a $2300 \mathrm{~mm}$. La parte más seca se ubica en el centro y sur de la entidad que comprende las áreas del Valle del Mezquital y Pachuca, cuyos valores de precipitación media anual están entre los 300 y $500 \mathrm{~mm}$. En la mesa central, la precipitación vuelve a aumentar hacia los límites con el Estado de México, con valores de 500 a $700 \mathrm{~mm}$ anuales e incluso con algunas áreas con precipitación media anual de hasta $800 \mathrm{~mm}$. La barranca de Metztitlán es otra zona con valores bajos de precipitación, con valores de 300 a $500 \mathrm{~mm}$ aumentando considerablemente la precipitación al ascender los vientos en las elevaciones montañosas de los municipios de Mineral del Chico y Mineral del Monte, alcanzando valores de más de $1000 \mathrm{~mm}$ (Tinoco, 2005).

Con la sobreposición de la cartografía resultante de las isoyetas e isotermas se obtuvo un mapa con 7981 áreas de influencia climática. Cada AIC se completó con su respectiva información de temperatura y precipitación mensual y anual, que posteriormente sirvió para ubicar las zonas de distribución potencial del cedro rojo.

\section{Aplicación de modelos de cambio climático}

Se sobrepuso el mapa de las AIC a la cartografía de cambios de temperatura y precipitación definidos por el Canadian Institute for Climate Studies (CICS, 2006) para los modelos GFDL-R30 y HadCM3,

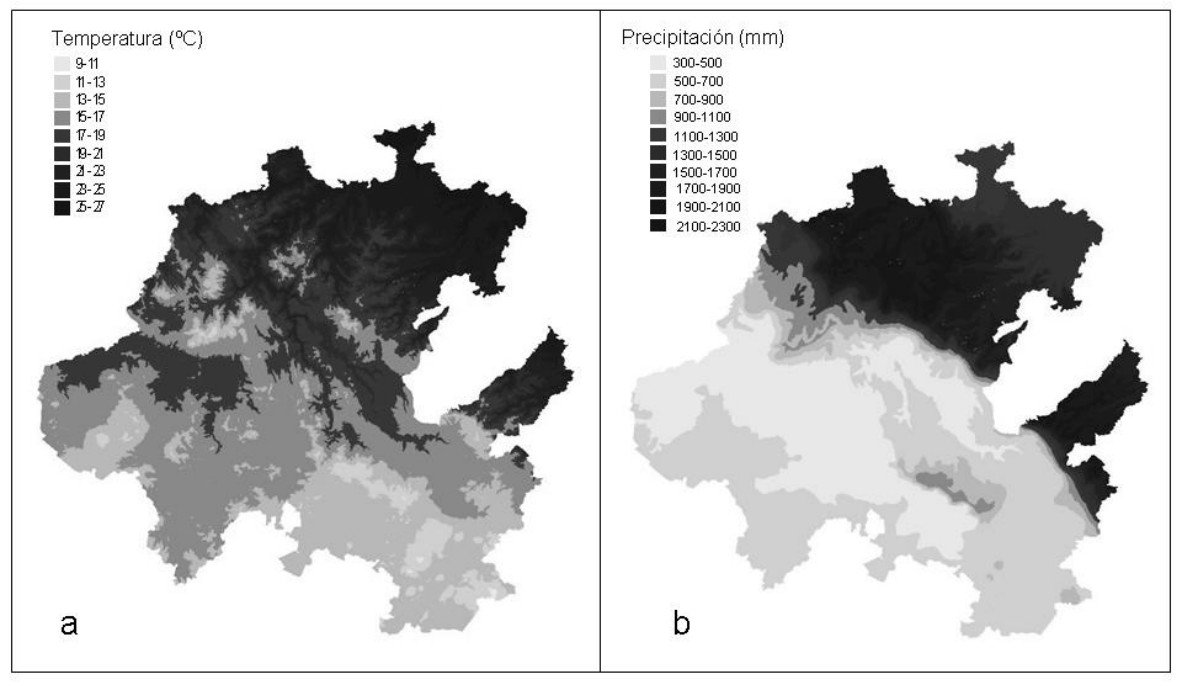

Figura 1. Mapas de temperatura y precipitación media anual del estado de Hidalgo: $a=$ temperatura media anual; $b=$ precipitación media anual. 
obteniéndose con esto las variables climáticas para los años 2020 y 2050 para el caso de los dos modelos utilizados.

Al aplicar los cambios a la temperatura y precipitación, se observa que de los dos modelos usados, el GFDL-R30 es el que pronostica un aumento en las dos variables climáticas (Figura 2 ). El valor promedio de incremento en toda la entidad para la precipitación media anual aplicando este modelo para los años 2020 y 2050 es de $100 \mathrm{~mm}$ y $120 \mathrm{~mm}$, respectivamente, esto comparado con el escenario base que es de $1300 \mathrm{~mm}$ anuales, el mayor aumento para los dos periodos se presenta en el mes de julio. El mes de octubre, por su parte, es en donde se observa la mayor disminución de la lluvia bajo este modelo. En cuanto a temperatura, los dos años simulados establecen un aumento en todos los meses, siendo el escenario para el 2050 en el que mayor incremento se observa, con un promedio anual de $1,7^{\circ} \mathrm{C}$ para todo el estado de Hidalgo a partir del valor del escenario base que es de una temperatura media anual de $17,9^{\circ} \mathrm{C}$ para todo el estado.

El modelo HadCM3 pronostica un aumento mayor de temperatura en toda la entidad con $1,1^{\circ} \mathrm{C}$ para el escenario del año 2020 y $2,5^{\circ} \mathrm{C}$ para el año 2050 (Figura 2). En cuanto a la precipitación se refiere, el modelo inglés establece una merma en la cantidad de lluvia promedio anual para los dos periodos con relación al escenario base de $137,8 \mathrm{~mm}$ para el año 2020 y 131,6 mm para el 2050 .

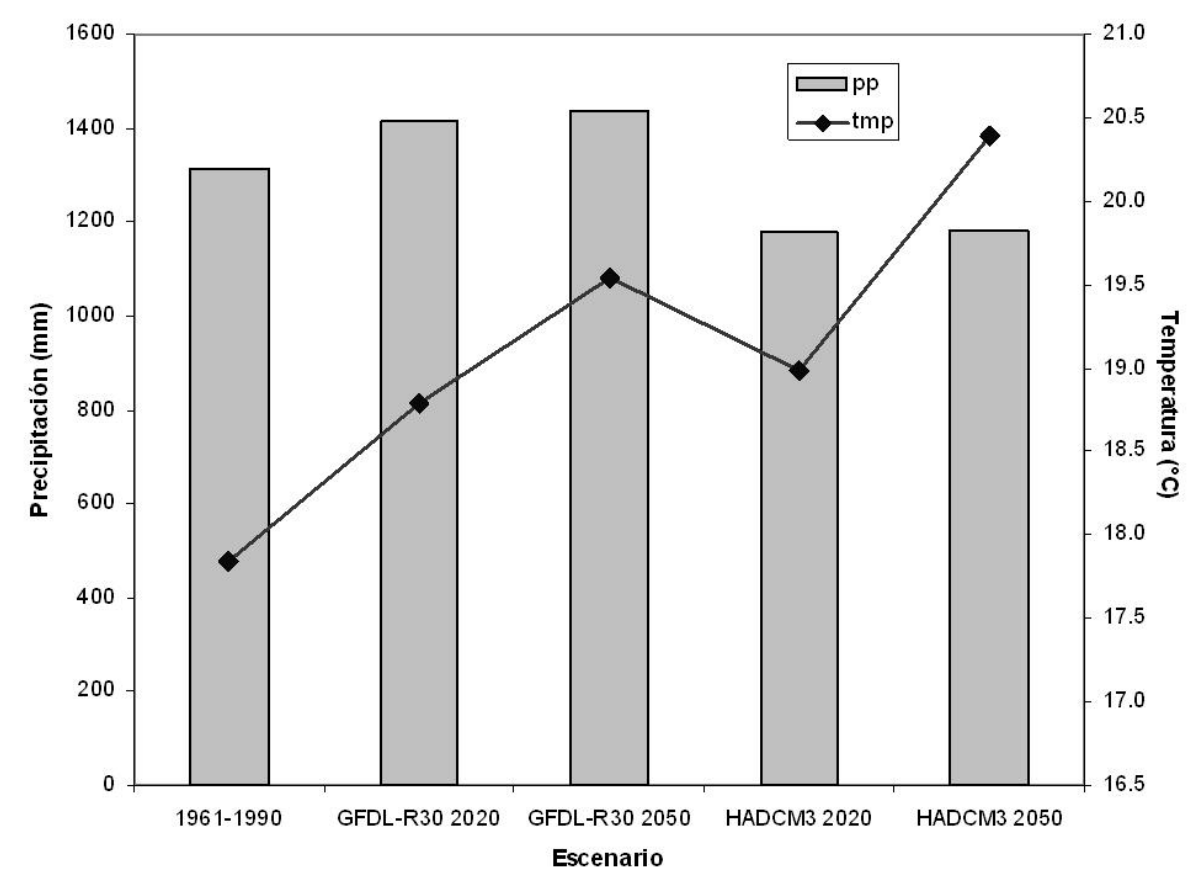

Figura 2. Temperatura y precipitación promedio anual para las condiciones actuales y los escenarios de cambio climático 


\section{Balance de humedad en el estado de Hidalgo}

En la Figura 3 se muestra la proporción de los tipos climáticos en los cinco escenarios evaluados. En el escenario actual (1961-1990), el tipo de clima que predomina es el Semiárido húmedo (D3) ocupando el $32,9 \%$ del territorio estatal, le sigue por cobertura el clima Húmedo (B) con el $19,6 \%$ y es seguido por el clima Per húmedo (A) con el 18,9\%. Los climas con menor cobertura fueron el Semiárido moderado (D2), Subhúmedo seco (C1) y el Subhúmedo húmedo (C2) con una cobertura del $14,9 \%, 9,6 \%$ y $4,2 \%$, respectivamente.

Los periodos simulados de cambio climático (2020 y 2050) con el modelo GFDL-R30 no presentan mucha diferencia con respecto a los climas del escenario base. Para el año 2020, el clima con mayor superficie es también el Semiárido húmedo con $32,7 \%$ de la superficie total del estado, seguido por el clima Húmedo con $21,7 \%$ y el clima Per húmedo con $13 \%$. Los climas C2, C1 y D2 ocupan cerca del $10 \%$ cada uno de ellos. Para el año 2050 la proporción es muy parecida al anterior escenario, donde el clima Semiárido húmedo ocupa la mayor superficie con $30,2 \%$, seguido del clima Húmedo con $22,1 \%$, el Per húmedo, Subhúmedo húmedo, Subhúmedo seco y el Semiárido moderado ocupan cerca del $10 \%$ cada uno.

Los resultados al aplicar el modelo HadCM3 muestran una disminución de los climas Per húmedo $(A)$ con respecto al escenario base y un incremento sustancial en el clima Semiárido moderado (D2). Para el año 2020 se pronostica que el clima Per húmedo ocupará el $4,1 \%$ del estado y el clima Húmedo el

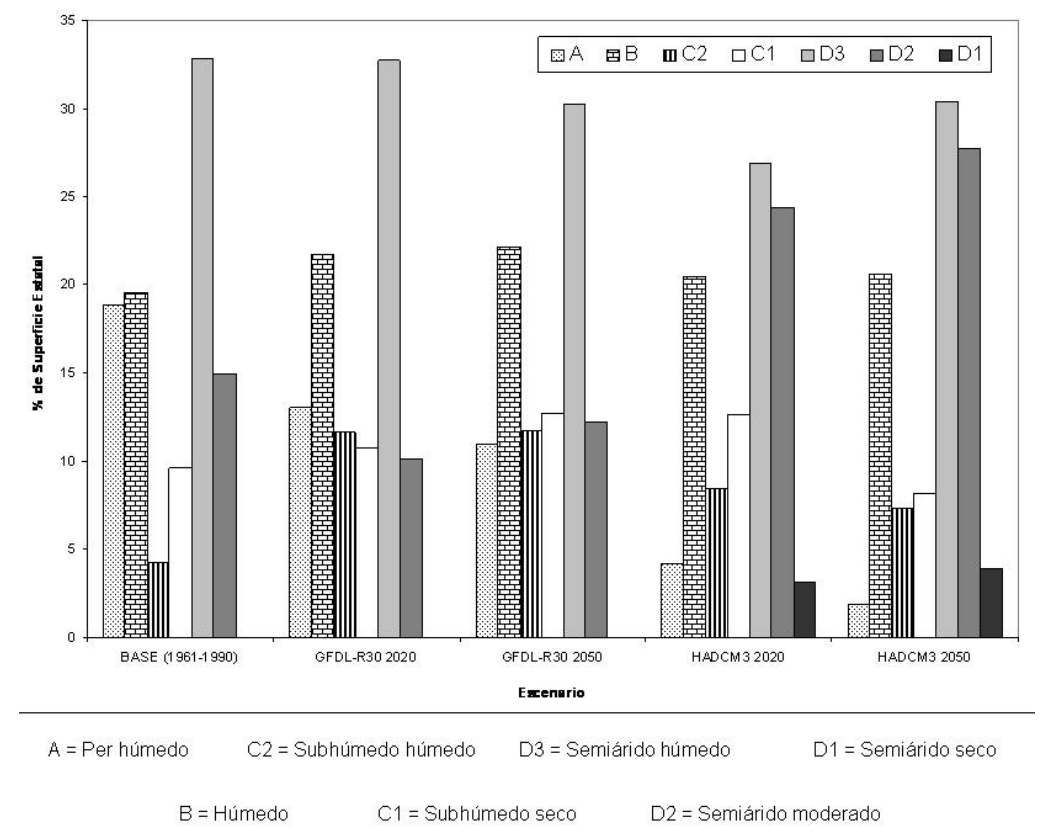

Figura 3. Distribución de los climas estimados con la metodología de Thornthwaite modificada versión III, bajo condiciones actuales y escenarios de cambio climático 
20,4\%. El clima Subhúmedo húmedo se pronostica en un $8,4 \%$ del territorio estatal, el Subhúmedo seco en el $12,6 \%$, el Semiárido húmedo en el $26,9 \%$ siendo también el de más amplia distribución, seguido del Semiárido moderado con $24,4 \%$. En el escenario del año 2050 , el clima A se pronostica en el $1,9 \%$ de la superficie estatal y el clima B se obtuvo para el $20,6 \%$. Los climas C2 y C1 disminuyen su cobertura en comparación con el periodo anterior al ubicarse en el 7,4\% y $8,1 \%$ de la superficie, respectivamente. Por otro lado, los climas D3 y D2 aumentan su superficie con respecto al escenario anterior, estimándose que ocuparán el $30,4 \%$ y $27,7 \%$, respectivamente.

En las simulaciones bajo el modelo inglés resalta la aparición del tipo de clima Semiárido seco (D1), ya que en los otros escenarios no se registra. Para el año 2020 este modelo establece que dicho clima se distribuirá en el $3,1 \%$ del estado y para el año 2050 en el 3,9\% de la superficie estatal.

\section{Distribución actual de Cedrela odorata L.}

En la Tabla 3 se muestra la compilación de los requerimientos climáticos del cedro rojo que se obtuvieron de las fuentes de información consultadas (CONAFOR, 2000; Vázquez et al., 1999).

La distribución actual del cedro rojo se presenta en la Figura $4 a$, misma que se obtuvo de la información del inventario nacional forestal (SEMARNAT-UNAM, 2001) relacionándola con la distribución de los tipos de vegetación con los que, de acuerdo a la CONAFOR (2000), se asocia esta especie. Estos tipos de vegetación fueron el bosque tropical perennifolio, el bosque tropical subcaducifolio, el bosque tropical caducifolio y el bosque mesófilo de montaña. La información obtenida muestra que el cedro rojo actualmente se encuentra distribuido en áreas fragmentadas a lo largo de la vertiente del Golfo de México, cubriendo una superficie de $2040,3 \mathrm{~km}^{2}$ lo que equivale al $9,8 \%$ del territorio total del estado de Hidalgo.

Tabla 3. Características climáticas y nivel de aptitud en las que crece el cedro rojo (Cedrela odorata L.)

\begin{tabular}{|c|c|c|c|c|}
\hline \multirow{2}{*}{ VARIABLE } & \multicolumn{4}{|c|}{ NIVEL DE APTITUD* } \\
\hline & 1 & 2 & 3 & 4 \\
\hline $\begin{array}{l}\text { Precipitación media } \\
\text { Anual }(\mathrm{mm})\end{array}$ & $2500-4000$ & $\begin{array}{l}4000-5000 \\
2000-2500 \\
\end{array}$ & $\begin{array}{c}>5000 \\
1200-2000 \\
\end{array}$ & $<1200$ \\
\hline $\begin{array}{l}\text { Duración del periodo } \\
\text { Seco (meses) }\end{array}$ & $0-3$ & $3-5$ & $5-6$ & $>6$ \\
\hline $\begin{array}{c}\text { Temperatura media } \\
\text { Anual }\left({ }^{\circ} \mathrm{C}\right)\end{array}$ & $20-26$ & $\begin{array}{l}26-28 \\
18-20\end{array}$ & $\begin{array}{l}28-30 \\
16-18\end{array}$ & $\begin{array}{l}>30 \\
<16\end{array}$ \\
\hline $\begin{array}{c}\text { Temperatura mínima } \\
\text { Promedio }\left({ }^{\circ} \mathrm{C}\right)\end{array}$ & $16-22$ & $\begin{array}{l}22-24 \\
14-16 \\
\end{array}$ & $\begin{array}{l}24-26 \\
11-14 \\
\end{array}$ & $\begin{array}{l}>26 \\
<11 \\
\end{array}$ \\
\hline $\begin{array}{l}\text { Temperatura máxima } \\
\text { Promedio }\left({ }^{\circ} \mathrm{C}\right)\end{array}$ & $24-30$ & $\begin{array}{l}30-34 \\
22-24\end{array}$ & $\begin{array}{l}34-38 \\
20-22\end{array}$ & $\begin{array}{l}>38 \\
<20\end{array}$ \\
\hline
\end{tabular}

Donde: $1=$ Apto, $2=$ Moderadamente Apto, $3=$ Marginalmente Apto y $4=$ No Apto

*Adaptado por los autores a partir de: CONAFOR (2000) y Vázquez et al. (1999). 


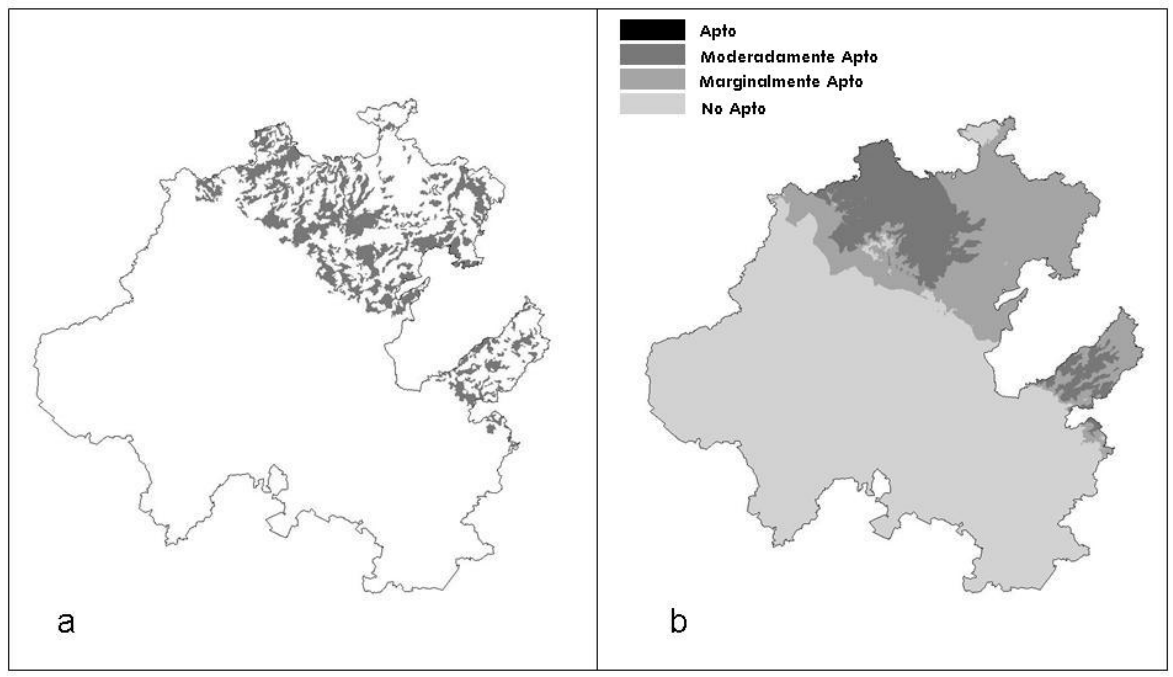

Figura 4. a= Distribución actual de los tipos de vegetación con los que se asocia Cedrela odorata L. y $b=$ niveles de aptitud de distribución potencial actual

\section{Distribución potencial actual de Cedrela odorata L. y bajo escenarios de cambio climático}

Aplicando los requerimientos climáticos del cedro rojo a la información actual de las áreas de influencia climática, se determinó la distribución potencial actual (Figura 4b), en donde se aprecia que el área con potencial climático para el desarrollo de la especie se encuentra ubicada en la zona de barlovento.

En la tabla 4 se presentan las superficies cubiertas por nivel de aptitud a partir de las variables climáticas para los cinco escenarios de acuerdo a los requerimientos de la especie expresados en la Tabla 3. El factor más restrictivo en todos los escenarios fue la precipitación media anual, no presentándose ninguna área con el nivel más alto de aptitud (Apto) para todos los escenarios estudiados. En los escenarios del modelo GFDL-R30 para este factor, en general aumenta la superficie con algún nivel de aptitud, presentándose un incremento con respecto al potencial actual de 472,84 y $628,64 \mathrm{~km}^{2}$ para los escenarios de $2020 \mathrm{y}$ 2050, respectivamente. Dentro de los niveles de aptitud, hay un incremento en la superficie para el nivel Moderadamente apto y una disminución para el nivel Marginalmente apto. En cambio para el modelo HadCM3, hay una disminución de la superficie con cierto grado de aptitud por este factor, con respecto al potencial actual, de 406,78 y $367,28 \mathrm{~km}^{2}$ para los escenarios de 2020 y 2050, respectivamente. La mayor parte de la superficie se ubica en la clase Marginalmente apto, y la disminución en la clase Moderadamente apto tiene valores de cero para el escenario del 2020 y de tan sólo 271,39 km² para el año 2050.

Para las variables asociadas a temperatura, en ambos modelos se presenta un aumento en la superficie con cierto grado de aptitud. Para los escenarios del GFDL-R30, el incremento en la superficie con aptitud con respecto al potencial actual del escenario base fue de 
Tabla 4. Superficie estimada por nivel de aptitud de Cedrela odorata L. de acuerdo a las variables climáticas para los cinco escenarios evaluados

\begin{tabular}{lcccc}
\hline & \multicolumn{4}{c}{ Superficie por Nivel de Aptitud $\mathbf{( k m}^{2}$ ) } \\
\cline { 2 - 5 } & Apscenario & $\begin{array}{c}\text { Moderadamente } \\
\text { Apto }\end{array}$ & $\begin{array}{c}\text { Marginalmente } \\
\text { Apto }\end{array}$ & No Apto \\
\hline & \multicolumn{4}{c}{ Precipitación } \\
\hline Potencial Actual & 0,00 & 2565,24 & 4202,83 & 14061,25 \\
GFDL-R30 al 2020 & 0,00 & 3959,92 & 3115,88 & 13588,40 \\
GFDL-R30 al 2050 & 0,00 & 4000,53 & 3231,06 & 13432,61 \\
HadCM3 al 2020 & 0,00 & 0,00 & 6196,18 & 14468,03 \\
HadCM3 al 2050 & 0,00 & 271,39 & 5964,32 & 14428,50 \\
\hline & \multicolumn{5}{c}{ Temperatura } \\
\hline Potencial Actual & 4573,10 & 2314,82 & 4334,73 & 9606,67 \\
GFDL-R30 al 2020 & 5081,62 & 4192,26 & 6470,19 & 4920,14 \\
GFDL-R30 al 2050 & 1495,63 & 3036,59 & 13438,01 & 2694,09 \\
HadCM3 al 2020 & 5081,62 & 4192,26 & 6470,19 & 4920,14 \\
HadCM3 al 2050 & 7456,29 & 7822,09 & 4576,06 & 811,76 \\
\hline & \multicolumn{5}{c}{ Periodo seco } \\
\hline Potencial Actual & 7313,99 & 1457,16 \\
GFDL-R30 al 2020 & 6690,21 & 8532,11 & 9993,17 & 2064,99 \\
GFDL-R30 al 2050 & 7092,55 & 5838,55 & 3391,65 & 2050,24 \\
HadCM3 al 2020 & 6494,89 & 2331,32 & 5044,28 & 2688,83 \\
HadCM3 al 2050 & 6307,68 & 5646,68 & 4034,62 & 6301,38 \\
\hline
\end{tabular}

4686,53 y $6912,58 \mathrm{~km}^{2}$ para el año 2020 y 2050, respectivamente. Para el modelo HadCM3, el incremento de la superficie con aptitud fue de 4686,53 y $8794,94 \mathrm{~km}^{2}$ para el año 2020 y 2050, respectivamente.

Para la variable duración del periodo seco que se estimó a través del balance de humedad entre la precipitación y la demanda evapotranspirativa, según el modelo GFDL-R30 no se tiene gran variación de la superficie con cierto grado de aptitud con respecto al escenario base. Hubo un ligero incremento de $14,59 \mathrm{~km}^{2}$ para el año 2020 y un decremento de $623,84 \mathrm{~km}^{2}$ para el 2050. En cambio, para el modelo HadCM3, para ambos escenarios se estimaron decrementos significativos de 4236,39 y $2610,24 \mathrm{~km}^{2}$ para el año 2020 y 2050, respectivamente.
La variable con mayor restricción determinó los niveles de aptitud, mismos que se presentan resumidos en la tabla 5 . La superficie potencial estimada bajo el escenario actual con categoría Moderadamente Apta se ubica en la parte noreste del estado, sobre las laderas intermedias de los flancos orientales de la Sierra Madre Oriental así como una pequeña porción en la zona sureste (Figura 4b), cubriendo $2169,80 \mathrm{~km}^{2}$, que corresponden al $10,5 \%$ del total de la superficie estatal. El nivel de aptitud Marginalmente Apto ocupa una superficie de $4102,73 \mathrm{~km}^{2}$, que corresponde al $19,86 \%$ del total de la superficie. El $69,65 \%$ de la superficie estatal corresponde al nivel No apto. 
Tabla 5. Superficie estimada por nivel de aptitud de Cedrela odorata L. para los cinco escenarios evaluados

\begin{tabular}{lcccc}
\hline \multirow{2}{*}{ Escenario } & \multicolumn{4}{c}{ Superficie por Nivel de Aptitud $\left(\mathbf{k m}^{2}\right)$} \\
\cline { 2 - 5 } & Apto & $\begin{array}{c}\text { Moderadamente } \\
\text { Apto }\end{array}$ & $\begin{array}{c}\text { Marginalmente } \\
\text { Apto }\end{array}$ & No Apto \\
\hline Potencial Actual & 0,00 & 2169,80 & 4102,73 & 14391,58 \\
GFDL-R30 al 2020 & 0,00 & 3708,86 & 3195,83 & 13759,52 \\
GFDL-R30 al 2050 & 0,00 & 2304,20 & 4879,91 & 13480,09 \\
HadCM3 al 2020 & 0,00 & 0,00 & 6091,38 & 14572,83 \\
HadCM3 al 2050 & 0,00 & 270,50 & 5962,97 & 14430,74 \\
\hline
\end{tabular}

En la figura 5 se muestran los resultados del comportamiento del cedro rojo aplicando los dos modelos de cambio climático. Para el modelo GFDL-R30, a nivel general se observa una disminución del nivel No Apto para ambos periodos $(2020$ y 2050 ) siendo de $66,59 \%$ y $65,23 \%$, respectivamente, en comparación del $69,65 \%$ que se tiene para el periodo potencial actual (1961-1990). En el nivel de Moderadamente apto, en ambos escenarios de cambio climático se tuvo un aumento, pasando de $10,5 \%$ en el escenario base a $17,95 \%$ para el año 2020 y a $11,15 \%$ en el 2050 . El Nivel Ligeramente apto disminuye para el escenario del 2020 con respecto al potencial actual pasando de $19,86 \%$ de la superficie a $15,47 \%$. Sin embargo, para el año 2050 aumenta a un $23,62 \%$ del total de la superficie. La distribución espacial de los diferentes niveles de aptitud con este modelo se muestra en la Figura 6a y 6b para el año 2020 y 2050, respectivamente.

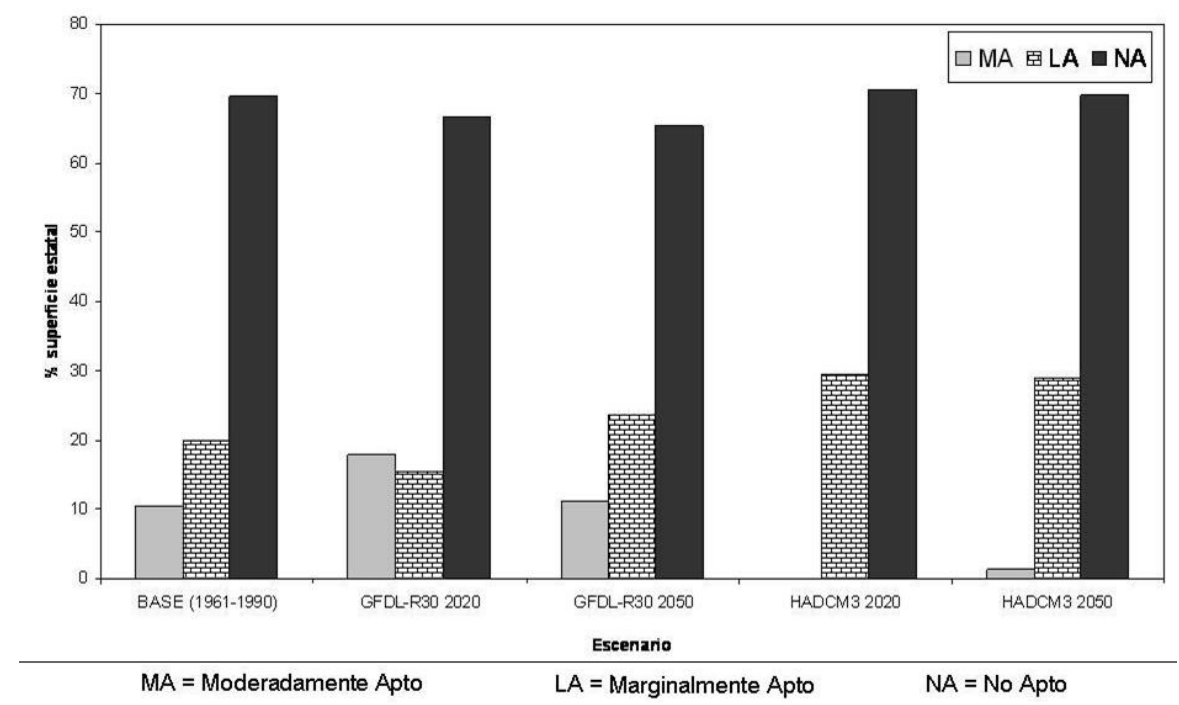

Figura 5. Cambios en los niveles de aptitud de Cedrela odorata L. bajo condiciones actuales y con escenarios de cambio climático 


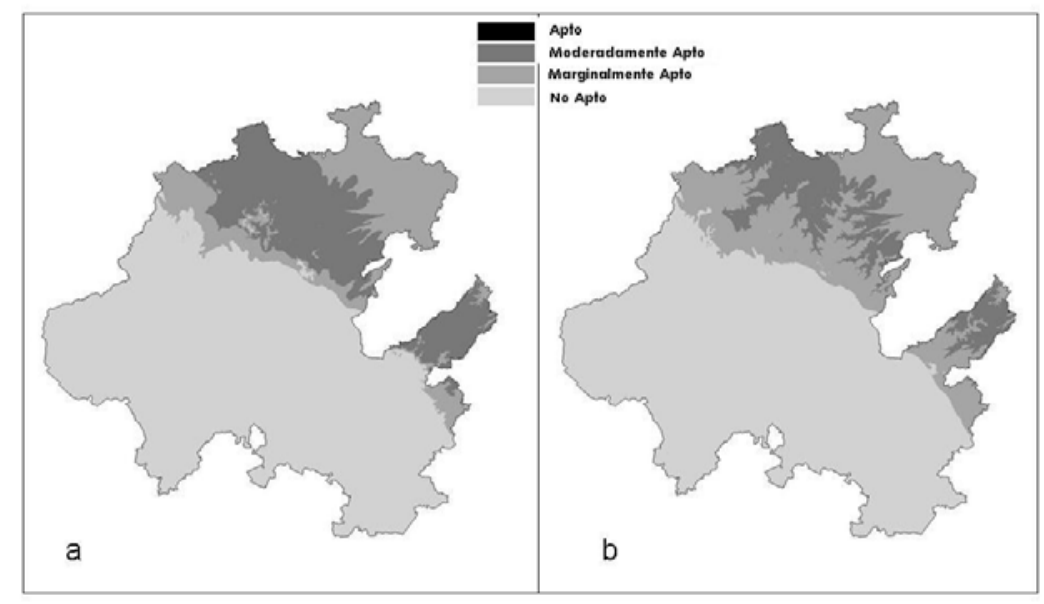

Figura 6. Distribución potencial de Cedrela odorata L. bajo escenarios de cambio climático estimados con el modelo GFDL-R30: (a) 2020; (b) 2050

El comportamiento del nivel de aptitud con el modelo HadCM3 (Figura 6), muestra un pequeño aumento en la superficie catalogada como No apta, con un porcentaje de la superficie estatal de $70,52 \%$ para el año 2020 y de $69,83 \%$ en el 2050. En la clase de Moderadamente apto, se da una gran disminución, pasando de $10,5 \%$ para el escenario potencial actual a cero en el año 2020 y $1,31 \%$ en el 2050. El Nivel Marginalmente apto, aumenta de $19,86 \%$ para el escenario base a $29,48 \%$ y $28,86 \%$ para los años 2020 y 2050 , respectivamente. La distribución espacial de los diferentes niveles de aptitud con este modelo se muestra en la Figura $7 \mathrm{a}$ y $7 \mathrm{~b}$ para el año 2020 y 2050 , respectivamente.

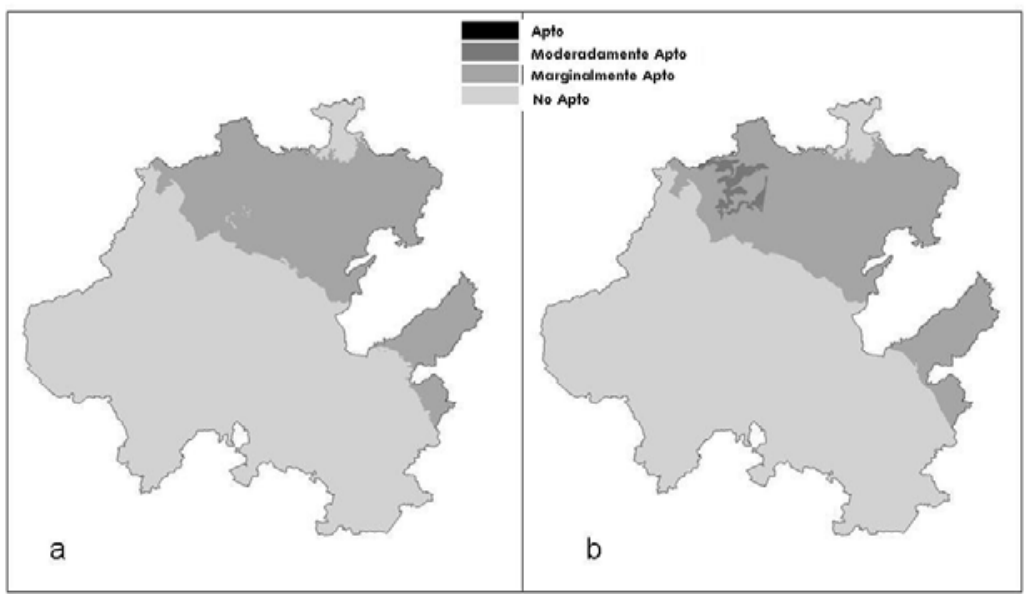

Figura 7. Distribución potencial de Cedrela odorata L. bajo escenarios de cambio climático estimados con el modelo HadCM3: (a) 2020; (b) 2050 


\section{DISCUSIÓN}

\section{Delimitación de áreas de influencia climática}

Los modelos de regresión lineal para las dos zonas presentan variaciones en el rango de incremento de la temperatura al aumentar la altura. La zona de barlovento, para todos los meses y el modelo anual, tiene rangos de variación altotérmica menores que la de sotavento. Esto se asocia al mayor contenido de vapor de agua del aire en esta zona, que al ascender sobre la pendiente, se presenta la condensación y la consecuente liberación de calor latente al pasar el agua de vapor a líquido, mismo que calienta el aire $y$ reduce el rango de enfriamiento (Arhens, 2003). Los coeficientes de regresión mayores para la zona de barlovento se asocian con el mayor rango de altura en que están localizadas las estaciones meteorológicas que se incluyen en los modelos (Gómez et al., 2006).

El trazo de las isoyetas mensuales muestra que la distribución de la precipitación en todo el estado es primordialmente en verano como resultado del efecto de los vientos alisios provenientes del Golfo de México, así como también de los huracanes que se forman en el Caribe y siguen su curso por el Golfo entre los meses de Julio a Octubre. Durante el invierno las lluvias son influenciadas por los frentes fríos llamados "nortes", mismos que están asociados al componente invernal del Monzón de Norteamérica, los cuales también aportan lluvia de forma considerable, principalmente en la zona de barlovento, aunque en menor cantidad que los sistemas de verano (Vidal, 2005).

Las zonas secas comprenden las áreas donde los vientos que pasan la barrera montañosa de la Sierra Madre Oriental al descender se comprimen, lo que ocasiona sombra de lluvia, y por lo tanto disminuye la precipitación. La precipitación aumenta de nuevo conforme las masas de vientos se retiran de las áreas de compresión y al desplazarse sobre valles altos donde la convección local es un elemento que aumenta el ascenso del aire y la formación de nubes (Mosiño y García, 1974). El aumento considerable de la precipitación en el macizo montañoso de Mineral del Chico y Mineral del Monte, se asocia con el ascenso forzado del aire sobre las laderas orientales de dichos sistemas montañosos y con la formación de nubes al enfriarse el aire por debajo del punto de condensación del vapor de agua y la formación de nubes, sobre todo cuando el gradiente de presión es considerable entre la zona del Golfo de México y el altiplano central que establece el flujo de los vientos alisios (Tinoco, 2005).

Aplicación de modelos de cambio climático. Ambos modelos pronostican un incremento de la temperatura, siendo mayor dicho aumento para el escenario del año 2050. El incremento de temperatura está ampliamente explicado por la acumulación de Gases de Efecto Invernadero (GEI) que, al ir aumentando su concentración en la atmósfera, causan una mayor retransmisión a la superficie de la radiación de onda larga irradiada por la superficie de la tierra, lo que calienta más el aire y provoca un incremento en la temperatura. Para el escenario más tardío se preve mayor acumulación de estos gases y la magnificación del fenómeno de calentamiento (IPCC, 2001).

El modelo GFDL-R30 pronostica un incremento menor de temperatura que el modelo $\mathrm{HadCM} 3$, con $0,95^{\circ} \mathrm{C}$ para el 2020 y $1,7^{\circ} \mathrm{C}$ para el 2050 comparados con $1,1^{\circ} \mathrm{C}$ y $2,5^{\circ} \mathrm{C}$ para estos años que se obtienen con el modelo inglés. Esta diferencia se asocia con las series de tiempo 
con las que se calibraron dichos modelos. El modelo norteamericano considera una cantidad significativa de estaciones de zonas con alta concentración de aerosoles, que son comunes en la costa este de dicho país, lo que reduce el calentamiento. En cambio, el modelo inglés presenta datos más globales donde estos elementos tienen menor peso (McGregor y Nieuwolt, 1998).

Las diferencias en precipitación estimadas en los escenarios de los modelos, indican que el modelo inglés reporta una disminución que se acentúa ligeramente al aumentar los años considerados. Esto se explica por un desplazamiento pequeño hacia el sur de la zona de corrientes descendentes y el menor avance de los centros de alta presión hacia el océano en la temporada caliente del año, lo que disminuirá la precipitación en las zonas aledañas al movimiento de estos sistemas. En cambio el modelo norteamericano pronostica una mayor evaporación sin que esta zona se vea afectada considerablemente por el movimiento de los sistemas anteriores, lo que trae consigo un incremento en la precipitación (McGregor y Nieuwolt, 1998).

\section{Balance de humedad en el estado de Hidalgo}

Ambos modelos reportan un incremento en el déficit de humedad, con el desplazamiento a tipos climáticos más secos. Este incremento es mayor para los resultados del modelo inglés, ya que en sus escenarios se estiman mayores incrementos en la temperatura, lo que aumenta la demanda evapotranspirativa, que aunado a la disminución de la precipitación, el balance de humedad presenta mayor déficit. En las estimaciones con el modelo norteamericano, aunque se preve que aumente la precipitación, esta no lo hace en la misma proporción que la demanda evapotranspi- rativa, y aunque el cambio en el balance de humedad es menos drástico que lo reportado en el otro modelo, también está presente un ligero aumento en el déficit y disminución de las demasías (Monterroso y Gómez, 2003).

\section{Distribución actual de Cedrela odorata L.}

Se observa que $C$. odorata $L$. es una especie característica de los ecosistemas definidos por los grupos climáticos del trópico húmedo y trópico subhúmedo. Dentro de los cuales se llega a encontrar tanto en las partes bajas de los bosques mesófilos de montaña, como en las partes bajas de los bosques de pino y en los bosques de pino-encino, además de los bosques tropicales perennifolio, subperennifolio, caducifolio y subcaducifolio. Presenta una distribución amplia en cuanto a precipitación media anual. Sin embargo en zonas con precipitaciones notablemente menores a $2500 \mathrm{~mm}$, la especie no se desarrolla favorablemente $y$ presenta fustes cortos y frecuentemente torcidos (Vázquez et al., 1999).

\section{Distribución potencial actual de Cedrela odorata L. y bajo escenarios de cambio climático}

La condición climática más limitativa es la precipitación media anual, ya que en el estado de Hidalgo no rebasa los 2500 $\mathrm{mm}$. Esto implica que no se encontrarán áreas con el nivel de aptitud mayor (Apta). Sin embargo, con los datos del escenario base se estima que aproximadamente el $30 \%$ de la superficie estatal tenga un cierto grado de aptitud para esta especie. La ubicación de estas áreas se presenta en la zona de barlovento, sobre parte de la planicie costera y las laderas orientales de la Sierra Madre Oriental así como en las cañadas que dan al Golfo de México. Los resultados de la aplicación 
del modelo GFDL-R30 indican un ligero incremento de un poco menos de $5 \%$ en la superficie con cierto grado de aptitud para esta especie, y se asocia con el incremento de la altura en la que puede prosperar el cedro al tenerse temperaturas más altas y el rango de precipitación y duración del periodo seco favorables dentro de estos niveles de aptitud.

Los escenarios del modelo HadCM3 reportan una disminución drástica en los niveles de aptitud, concentrándose toda la superficie en prácticamente el nivel más bajo (Marginalmemnte apto); aunque la proporción de la superficie no apta permanece casi sin cambio respecto al escenario base. Sin embargo, en las zonas con menor altitud, mismas que les corresponden las temperaturas más altas con el incremento estimado, la evapotranspiración potencial se incrementa exponencialmente, determinando que en el balance de humedad la duración del periodo seco se incremente, pasando estas áreas a ser no aptas para la especie (Figura 7a y 7b). En los escenarios resultantes de este modelo también se tiene un desplazamiento a mayor altura de la cota de áreas con cierto grado de adaptabilidad en la zona de barlovento.

\section{CONCLUSIONES}

Aunque actualmente se reporta que el $9,8 \%$ de la superficie del estado presenta los tipos de vegetación con los que se asocia Cedrela odorata L., las estimaciones a partir de las características climáticas del escenario base (19611990), muestran que el $30,4 \%$ del estado presenta algún grado de aptitud para el desarrollo de esta especie. Las áreas con aptitud se ubican fundamentalmente en la zona de barlovento, que comprende la porción de la planicie costera del Golfo, el flanco este de las laderas de la Sierra Madre Oriental y las cañadas que desembocan a la planicie costera.
Los escenarios de cambio climático estimados con los modelos GFDL-R30 y el HadCM3 muestran una variabilidad en la distribución del cedro rojo. Ambos modelos pronostican un aumento en la temperatura, lo que incrementa la altura (sobre el nivel del mar) del área con aptitud en las laderas orientales de la Sierra Madre. En cambio, el modelo norteamericano pronostica un incremento menor de temperatura y un aumento ligero en la precipitación, lo que aumenta el área con algún nivel de aptitud con respecto al escenario base actual, con un $3,1 \%$ para el año 2020 y de $4,4 \%$ para el año 2050. Sin embargo, este aumento es diferencial dentro de las clases de aptitud, para el primer periodo la clase Moderadamente apta aumenta de $10,5 \%$ en el escenario base a $18,0 \%$ para el año 2020, pero para el escenario de 2050 disminuye a $11,2 \%$ que, aunque mayor que el escenario base, el incremento es reducido.

Bajo el modelo HadCM3 la distribución del cedro rojo se ve afectada. Esta se desplaza de la zona baja de la planicie costera a rangos de mayor altitud sobre las laderas del flanco este de la Sierra Madre Oriental. La superficie con nivel de aptitud disminuye 0,9\% para el año 2020 y $0,2 \%$ para el año 2050 . Sin embargo, el nivel de Moderadamente apto pasa de $10,5 \%$ en el escenario base a $0 \%$ para el año 2020 y a $1,3 \%$ para el año 2050 , ubicándose practicamente todas las áreas en el menor nivel de aptitud.

Los resultados diferentes de los dos modelos de cambio climático resaltan lo complejo de estos estudios, en donde existe un rango amplio de incertidumbre que aún se tiene ante cambios en los componentes climáticos globales y regionales. Al ser dos escenarios que señalan niveles muy amplios de comportamiento futuro, éstos deben tomarse como un primer acercamiento en la evaluación de 
los posibles impactos de un cambio climático en la especie y su ecosistema. Por ello, es menester que se realicen estudios más detallados donde se evalúe localmente a la especie. Sin embargo, los resultados aquí presentados señalan que independientemente del escenario que se tome, se observan posibles cambios en el comportamiento de la especie, por lo que deben incorporarse acciones inmediatas dentro de los programas regionales de manejo y conservación de la especie.

Las regiones que deben ser sujetas de monitoreo son aquellas donde el cedro rojo podría dejar de tener las condiciones favorables para su desarrollo, ya que estas son las zonas más vulnerables para la especie al mostrar potenciales de cambio.

En este contexto, se requieren estudios futuros a escala regional y local donde se involucre a campesinos, tomadores de decisiones, dueños de la tierra y gobiernos en sus distintos niveles, para que en conjunto se desarrollen acciones y planes de adaptación ante el cambio climático.

\section{REFERENCIAS}

ACIA. 2004. Impacts of a warming Artic. Artic Climate Impact Assessment. Cambridge University Press. Cambridge. 146 p.

Aguilera, M. y R. Martínez. 1996. Relaciones agua-suelo-planta-atmósfera. Universidad Autónoma Chapingo. México. 256 p.

Ahrens, C. D. 2003. Meteorology today: an introduction to weather, climate, and the environment. $7^{\text {th }}$ ed. Brooks Cole. Pacific Grove, CA; EUA. 608 p.

Bakkenes, M., B. Eickhout y R. Alkemade. 2006. Impacts on different climate stabilization scenarios on plant species in Europe. Global Environmental Change 16:19-28.

Beaumont, L., L. Hughes y M. Poulsen. 2005. Predicting species distribution: use of climatic parameters in BIOCLIM and its impacts on predictions of species current and future distributions. Ecological Modelling 186(2):251-270.

Berry, P., T. Dawson, P. Harrison y R. Pearson. 2002. Modeling potential impacts of climate change on the bioclimatic envelope of species in Britain and Ireland. Global Ecology and Biogeography 11:453-462

CICS (Canadian Institute for Climate Studies), URL: www.cics.uvic.ca, (15/Feb/2006).

CONAFOR. 2000. Ficha técnica de Cedrela odorata L., Comisión Nacional Forestal. México. $6 \mathrm{p}$.

Dunne, T. y L. Leopold. 1978. Water in environmental planning. Ed. W. H. Freeman. EUA. $818 p$.

ESRI. 1996. Environmental Systems Research Institute, Inc., 380 New York Street. Redlands. CA. 92373. EUA.

Gómez, D.J.D., J. Etchevers, A. Monterroso, C. Gay, J. Campo y M. Martínez. 2006. Spatial estimation of mean temperature and precipitation in areas of scarce meteorological information. En revisión por Atmósfera.

Herrmann, S., A. Anyamba y C. Tucker. 2005. Recent trends in vegetation dynamics in the African Sahel and their relationship to climate. Global Environmental Change 15(4):394404. 
Hitz, S. y J. Smith. 2004. Estimating global impacts from climate change. Global Environmental Change 14(3): 201-218.

Houghton, J.T., L.G. Meira Filho, B.A. Chander, N. Harris, A. Kattenberg y K. Maskell (Eds). 1996. Climate Change 1995: The science of climate change. Cambridge University Press, Cambridge.

IPCC (Intergovernmental Panel on Climate Change). 2001. Tercer informe de evaluación. Cambio climático 2001: la base científica. Cambridge University Press. Cambridge. 94 p.

IPCC (Intergovernmental Panel on Climate Change). 2002. Cambio climático y biodiversidad. Documento técnico $\mathrm{V}$ del IPCC. Cambridge University Press. Cambridge. 93 p.

Magaña, V., C. Conde, O. Sánchez y C. Gay. 2000. Evaluación de escenarios regionales de clima actual y de cambio climático futuro para México. In: C. Gay, ed. 2003. México: Una visión hacia el siglo XXI. El cambio climático en México. Instituto Nacional de Ecología, Universidad Nacional Autónoma de México, U.S. Country Studies Program, México. 18 p.

McGregor, G.R., y S. Nieuwolt. 1998. Tropical climatology: an introduction to the climate of the low latitudes. Second edition. John Wiley and Sons, Inglaterra. 352 p.

Monterroso Rivas, A.I. y J.D. Gómez Díaz. 2003. Escenarios climatológicos de la República Mexicana ante el cambio climático. Comisión Nacional de Zonas Áridas y Universidad Autónoma Chapingo, México. 170 p.
Mosiño, P.A. y E. García. 1974. The climate of México. In: R.A. Bryson and F. K. Hare, Eds. World Survey of Climatology, Vol. II, Climates of North America, Elsevier, p. 373-404.

Olfert, O. y R.M. Weiss. 2006. Impact of climate change on potential distributions and relative abundances of Oulema melanopus, Meligethes viridescens and Ceutorhynchus obstrictus in Canada. Agriculture, Ecosystems and Environment 113(14):295-301.

Ortiz Solorio, C.A. 1987. Elementos de agrometeorología cuantitativa con aplicaciones en la República Mexicana. Universidad Autónoma Chapingo, México. 327 p.

Pérez, P. 2005. Introducción a la biogeografía. Universidad Autónoma Chapingo, México. 237 p.

Pounds, J., M. Bustamante, L. Coloma, J. Consuegra, M. Fogden, P. Foster, E. La Marca, K. Masters, A. Merino, R. Puschendorf, S. Ron, G. Sanchez, C. Still y B. Young. 2006. Widespread amphibian extinctions from epidemic disease driven by global warming. Nature 439(12):161-167.

Rivera, M. 1999. El cambio climático. Consejo Nacional para la Cultura y las Artes, México.

SEMARNAT-UNAM. 2001. Inventario nacional forestal de la República Mexicana. Secretaría de Medio ambiente y Recursos Naturales, Universidad Nacional Autónoma de México, México.

SMN (Servicio Meteorológico Nacional). 2000. Normales climatológicas de la República Mexicana. Servicio Meteorológico Nacional, México. 
Tinoco, J. A. 2005. Identificación y evaluación de patrones de distribución de 3 especies forestales en el estado de Hidalgo, su distribución actual y frente al cambio y vulnerabilidad climática. Tesis de licenciatura, Universidad Autónoma Chapingo, México. 160 p.

Vázquez, C., A. Batis, M. Alcocer, M. Gual y C. Sánchez. 1999. Árboles y arbustos potencialmente valiosos para la restauración ecológica y la reforestación. Reporte técnico del proyecto J084. CONABIO - Instituto de Ecología, UNAM, México.

Velázquez, J. 2002. Agrofenoclimatología. Universidad Autónoma del Estado de México, México. 338 p.
Vidal, R. 2005. Las regiones climáticas de México. Textos monográficos: 2 Naturaleza. Instituto de Geografía, UNAM, México. 213 p.

Yánez, A., R. Twilley y A.L. Lara. 1998. Los ecosistemas de manglar frente al cambio climático global. Madera y Bosques 4(2):3-19.

Zunino, M. y A. Zullini. 2003. Biogeografía, la dimensión espacial de la evolución. Fondo de Cultura Económica, México. 359 p.

Manuscrito recibido el 31 de agosto de 2006

Este documento se debe citar como:

Gómez Díaz, J.D., A. I. Monterroso Rivas y J. A. Tinoco Rueda. 2007. Distribución del cedro rojo (Cedrela odorata L.) en el estado de Hidalgo, bajo condiciones actuales y escenarios de cambio climático. Madera y Bosques 13(2):29-49 
